\title{
Editorial
}

\section{Nonperturbative Approaches in Field Theory}

\author{
Ralf Hofmann $\mathbb{D}^{1},{ }^{1}$ Thierry Grandou $\mathbb{D}^{2},{ }^{2}$ and Mario Gattobigio ${ }^{2}$ \\ ${ }^{1}$ Universität Heidelberg and Karlsruhe Institute of Technology, Heidelberg, Eggenstein-Leopoldshafen, Germany \\ ${ }^{2}$ Institut de Physique de Nice, Sophia Antipolis, France
}

Correspondence should be addressed to Ralf Hofmann; ralf.hofmann2@kit.edu

Received 26 April 2018; Accepted 26 April 2018; Published 29 May 2018

Copyright (c) 2018 Ralf Hofmann et al. This is an open access article distributed under the Creative Commons Attribution License, which permits unrestricted use, distribution, and reproduction in any medium, provided the original work is properly cited. The publication of this article was funded by SCOAP $^{3}$.

Perturbation theory has long been the method of choice in addressing the $S$-matrix and related observables predicted by quantum field theories subject to well defined asymptotic scattering states and an accordingly local and sufficiently weak interaction, suggesting an analysis in terms of powers of a small coupling. Gauge theories like Quantum Electrodynamics (QED), the electroweak sector of the Standard Model (SM) of particle physics as a whole, and the primary perturbative vertices of Quantum Chromodynamics (QCD) describing, e.g., the creation of jets of hadrons from electronpositron annihilation at large center-of-mass energies thanks to asymptotic freedom are empirically very successful applications of this approach. Among many other important results, they gave us a quantitative description of radiative effects such as the deviation of the electron's spin induced magnetic moment (the $g$-factor) from the value predicted by the Dirac equation, a quantitative explanation of the Lamb shift, and the precise evolution of the strength of the electromagnetic and strong couplings $\alpha$ and $\alpha_{s}$ in dependence of the momentum transfer in given scattering processes. On the conceptual side, a major accomplishment of the perturbative approach is the demonstration by 't Hooft and Veltman of renormalizability of quantum gauge theory in four space-time dimensions with and without spontaneous gauge-symmetry breaking through a (renormalizable) Higgsfield sector, implying the intactness of the predictivity of these theories under the renormalization programme: the subtraction of loop-inherent infinities to dress only the parameters of the tree-level theory under physically imposed conditions and this to all loop orders. The thus enabled precise predictions of electroweak observables in the SM were impressively confirmed by experiments performed at the Large Electron-Positron Collider (LEP).
A genuine testing of the $\mathrm{U}(1) \times \mathrm{SU}(2) \times \mathrm{SU}(3)$ gauge dynamics of the perturbatively understood SM beyond perturbation theory must, however, avoid model assumptions such as suggestively parameterized parton distribution functions and the associated factorization of soft-momentum, nonperturbative contributions to hadronic polarization tensors. One usually argues that lattice gauge theory-a firstprinciple approach to QCD based on an ultraviolet (UV) and infrared (IR) cutoff formulation on a finite 4D Euclidean space-time lattice-produces results which do not rely on such assumptions and this increasingly well as computational resources become more and more abundant and the available conceptual and computational techniques more and more refined. But even for the pure Yang-Mills case (no fermions which couple to the gauge fields), there are various issues inherent to this approach: (i) problems in the direct simulation of dynamical processes (form factors at timelike momentum transfer, certain decay amplitudes) due to formulation of lattice gauge theory in Euclidean space-time, (ii) finite-size scaling at finite temperature and its profound impact on infrared sensitive thermodynamical quantities, and (iii) customary simulations of the Euclidean YangMills partition function subject to the Wilson discretization of the fundamental Yang-Mills action with the UV cutoff dependence (lattice spacing) and coarsening effects usually parameterized in terms of the perturbative Yang-Mills $\beta$ function. A nonperturbative, perfect quantum lattice action by definition does not exhibit any cutoff dependence at finite lattice spacing. It is, however, prohibitively expensive to construct by successions of numerical block-spin transformations. Apart from these technical problems, the determination of static observables (which do not depend on the Euclidean formulation), like the mass spectrum of light 
hadrons, is the result of black-box numerical experiments and as such does not openly provide insights into the underlying physics of ground-state structure, confinement, chiral symmetry breaking, and excitability. There is activity in lattice gauge theory, however, which poses these questions about mechanisms directly, thus providing valuable guidance to analytical approaches to nonperturbative Quantum YangMills theory.

Some of the contributions to this present special issue focus on just such nonperturbative approaches to pure Yang-Mills theory, QCD, and Yang-Mills thermodynamics. Namely, the work of $\mathrm{H}$. Reinhardt et al. investigates the nonperturbative vacuum functional in the Hamiltonian formulation of SU(2) and SU(3) QCD in Weyl-Coulomb gauge both at finite and at zero temperature and for both situations, pure Yang-Mills and fluctuating quark fields. The task is to solve the functional Schrödinger equation with a duly gaugefixed Hamiltonian for the ground-state functional. This is done in an approximate form in terms of minimizing energy in a well-motivated Gaussian-variational ansatz (and cubic plus quartic generalizations thereof) for the ground-state functional. The associated kernels can be expressed as solutions to Dyson-Schwinger and to gap equations which can be solved subject to simplifying assumptions and boundary (horizon) conditions to implement confinement (a highly nonperturbative feat). Interesting quantities to compute are the Wilson and Coulomb string tension. One then is led to ask about the field configurations that mainly induce them. This is done in comparison of the present continuum approach to lattice results in discussing the role of centervortex loops and the magnetic monopoles that associate with them. Finite temperature is introduced by compactification of the spatial dimension after $O(4)$ rotation, and the Polyakov loop as an order parameter for deconfinement is computed with the according ground-state functional used to compute the expectation of the $A_{0}$ field in Polyakov gauge. Again, the results are compared to lattice results yielding a good agreement. Approaches like the one pursued by $\mathrm{H}$. Reinhardt et al. in this special issue have helped to elucidate a number of important nonperturbative mechanisms in non-Abelian gauge theory. Exemplarily, we now sketch the content of some of the papers.

There are two interesting contributions in this special issue, one by S. J. Brodsky and the other one by H. G. Dosch, who base their nonperturbative approach to QCD on the so-called AdS-QCD conjecture which is believed to be related to the AdS-CFT holography correspondence, conjectured by J. Maldacena to hold between $4 \mathrm{D} \mathrm{SU}(N \rightarrow$ $\infty), \mathcal{N}=4$ SUSY Yang-Mills theory, and the corresponding 5D supergravity dual theory on anti-de-Sitter background space-time $\left(\mathrm{AdS}_{5}\right)$. In particular, S. J. Brodsky first introduces a mass scale into the QCD light-front Hamiltonian by adding a term proportional to the special conformal operator which then gives rise to a confining potential. This does not break conformal invariance on the level of the QCD action. This somewhat ad hoc procedure can be shown to follow from the conjectured duality between light-front QCD and $\mathrm{AdS}_{5}$ if one introduces the $\mathrm{AdS}_{5}$ action by the dilaton along the fifth dimension. Generalizing this by appealing to the full superconformal algebra, Brodsky obtains, among interesting insights about the light-front vacuum structure, a unified hadron spectroscopy for mesons, baryons, and tetraquarks with supersymmetric relations between meson and baryon masses. H. G. Dosch (in collaboration with S. J. Brodsky and G. de Teramont) makes the case for supersymmetry across the light hadron spectrum very explicit in appealing to the superconformal algebra to fix the form of the lightfront potential. On the other hand, heavy-quark symmetry seems to ensure the survival of supersymmetry even though conformal symmetry is strongly broken in the heavy-quark limit.

A long-standing question about the asymptotic behavior of perturbative expansions, here in QED, is pursued by I. Huet et al. There are good, classical arguments that this expansion does not converge. However, in particular, the leading large$N$ photon amplitude in the effective Euler-Heisenberg (E-H) theory can be constructed from a conjectured all-loop expression of the imaginary part of the E-H Lagrangian for scalar and spinor QED by Borel dispersion, suggesting the usefulness of a perturbative approach in expanding this amplitude in powers of $\alpha$ in a convergent way. However, an explicit example in $1+1$ dimension does not support such a possibility.

P. Mathieu discusses the possibility of performing exact nonperturbative computations of functional integrals related to the partition function and observables in 3D U(1) ChernSimons theory thanks to the Deligne cohomology classes of its fiber bundles.

$\mathrm{H}$. Weigel reports about his development of an efficient method, based on scattering data (spectral method) about a scalar field configuration of given topology (static solution to the classical equation of motion), to compute its one-loop effective potential (or vacuum polarization energy, VPE) in a $1+1 \mathrm{D} \phi^{6}$ (nonrenormalizable) theory where fluctuations do not naturally decompose into parity eigenstates since the background field may connect inequivalent vacua (not related by a parity transformation). Still, at the one-loop level, the VPE can be fixed unambiguously by UV subtractions. As a consequence, such a soliton may exhibit a VPE which no longer exhibits the breaking of translational invariance it had introduced on the classical level. This is an interesting, quantum induced, emergent phenomenon. Quantifying VPEs for even more general potentials may turn out to be insightful regarding quantum effects in soliton binding.

T. Krajewski presents a beautiful discussion on an extension of validity of the Wigner semicircle law for the probability distribution of eigenvalues of $N \times N$ Hermitian, independent Gaussian, or Wigner random matrices (offdiagonal entries independent and identically distributed with zero mean, diagonal entries identically distributed with finite mean and independent of off-diagonal entries) in the large$N$ limit to the case of correlated entries. Namely, assuming a certain scaling bound for the cumulants of entries, he shows by means of a renormalization-group equation for the effective action of the replica that bounds on effective cumulants are implied. In the large- $N$ limit, this, in turn, guarantees that only Gaussian terms contribute such that one is back at the semicircle law. Krajewski's work is an important contribution 
in showing the universality of eigenvalue distributions in a large class of high-dimensional random matrices.

The contribution by J. L. Rubin is a bit off the main theme of the special issue, yet highly interesting: insights derived from relativistic positioning systems for the structure of space-time. Namely, he shows how causal axiomatics and certain local $1 \mathrm{D}$ and $2 \mathrm{D}$ projective structures attached to emitters are sufficient to deduce the 4D projective structure of space-time. This allows, e.g., for a modification of Newton's force law on large distances.

M. Faber in his paper proposes a model of the electron along the lines of a Skyrme-model-like construction (but with a different potential term). The basic quantity, a spatial dreibein, parameterizes unit quaternions, that is, group elements of SU(2) with nontrivial winding on its group manifold $\mathrm{S}_{3}$ which give rise to a connection, in turn, defining the curvature tensor. Solving the static field equations, this yields solitons whose topological charge can be matched to electric charge after a reduction of the non-Abelian curvature to the 't Hooft tensor is performed. The extent of this static field configuration essentially matches the classical electron radius. There are two more topological quantum numbers which relate to spin and photon number. The model allows for magnetic currents which should make this description of classical electrodynamics nonlocal. The ideas presented here are very interesting because they may help to demystify the electron. It would be welcome if the emergence of the Compton wavelength, a more intuitive grasp of electron spin, and the apparent pointlikeness of the electron in highenergy scattering experiments could be understood, possibly in extensions of the model incorporating quantum effects from the outset and doing justice to L. de Broglie's work on the thermodynamics of the isolated particle.

The paper by T. Grandou et al. addresses an effective formulation of QCD which exhibits locality in fermionic Green's functions upon a sophisticated and highly nonperturbative functional integration (actually, functional differentiation) of gluonic gauge-field fluctuations, using the Halpern quadrature of the QCD partition function for the gauge field $A_{\mu}$. In the present paper, the emergence of gauge-fixing independence (the fundamental formulation of the QCD partition function is subjected to gauge fixing) of nonperturbative, fermionic Green's functions is discussed in a sequence of relaxed high-energy approximations (quenching, eikonal, Fradkin's representation of fermionic Green's functions and, finally, no Fradkin's representation) to scattering of quarks off quarks. This result is put into perspective by a very instructive comparison with conventional, perturbatively minded approaches to quark scattering in QCD and electron scattering in QED.

Finally, there are two papers, one by I. Bischer and one by S. Hahn and R. Hofmann, which explore consequences of deconfining SU(2) Yang-Mills thermodynamics. The former contribution addresses certain (nonperturbative) radiative corrections to the pressure, arising from massive quasi-particle fluctuations. It is shown that fixed-order dihedral diagrams exhibit a high-temperature dependence starkly exceeding the Stefan-Boltzmann behavior. However, it is demonstrated that an all-order resummation cures this apparent problem and leads to well-bounded, purely imaginary contributions at leading order (implying that these radiative corrections do not admit a thermodynamical interpretation). The second paper investigates the consequences of the postulate that an $\mathrm{SU}(2)$ rather than a $U(1)$ gauge principle governs thermal photon gases, e.g., the Cosmic Microwave Background (CMB), for the high- $z$ cosmological model. Specifically, a modification of the $T-z$ relation implies that the high- $z$ cosmological model has to contain less dark matter than the $\Lambda C D M$ standard model posits. This is argued to resolve the tension in $H_{0}$ between fits to the power spectra of the $\mathrm{CMB}$ and its cosmologically local extraction.

All papers of this special issue have undergone peer review by one or two high-calibre referees. A substantial fraction of manuscripts submitted had to be rejected.

Ralf Hofmann Thierry Grandou Mario Gattobigio 

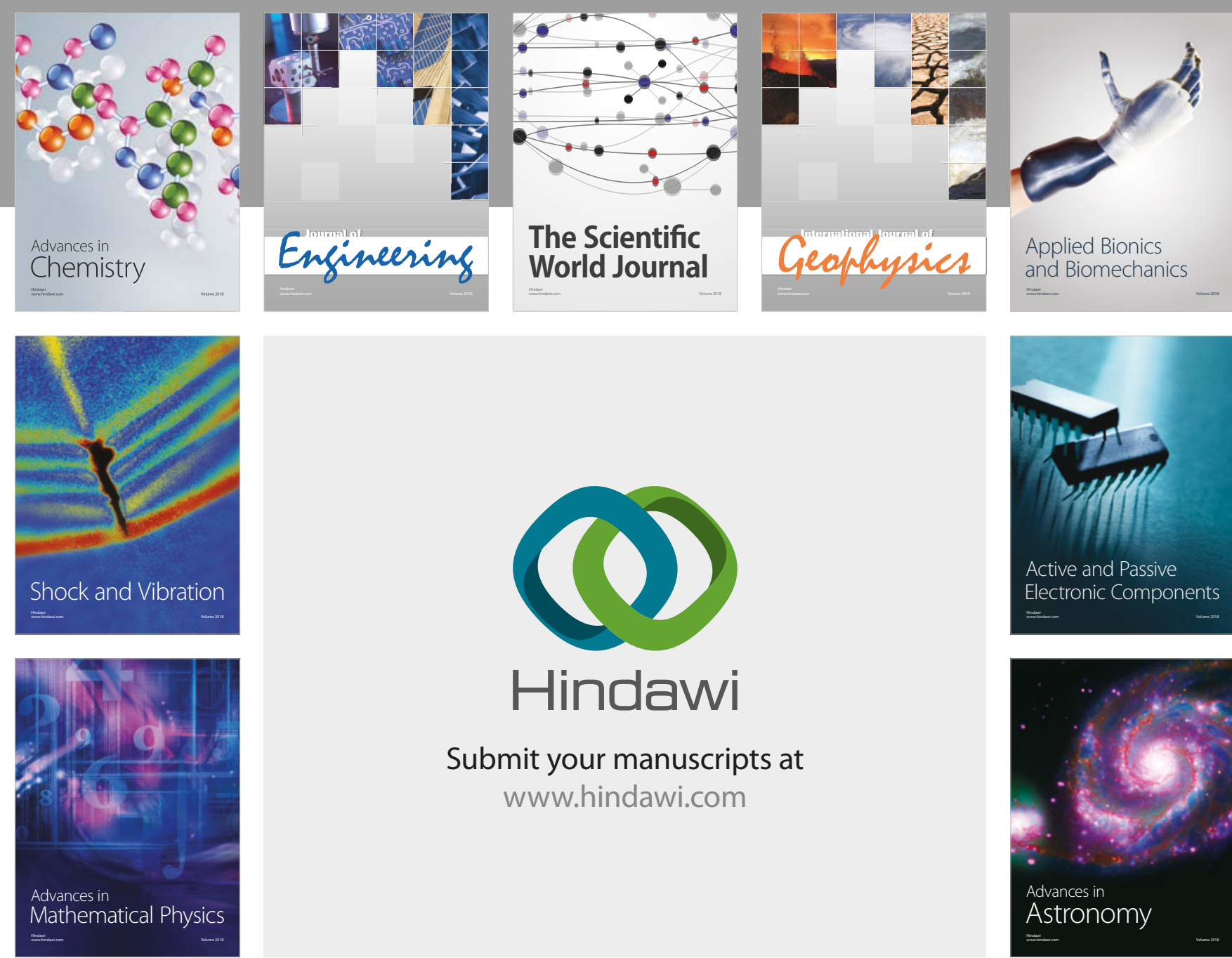

Submit your manuscripts at

www.hindawi.com

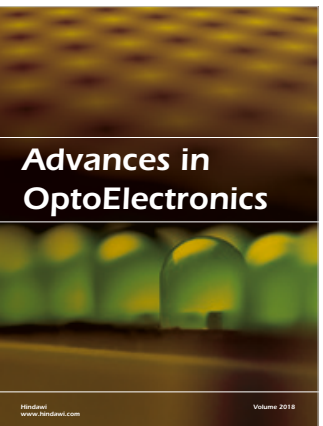

\section{Rotcting Machinery}
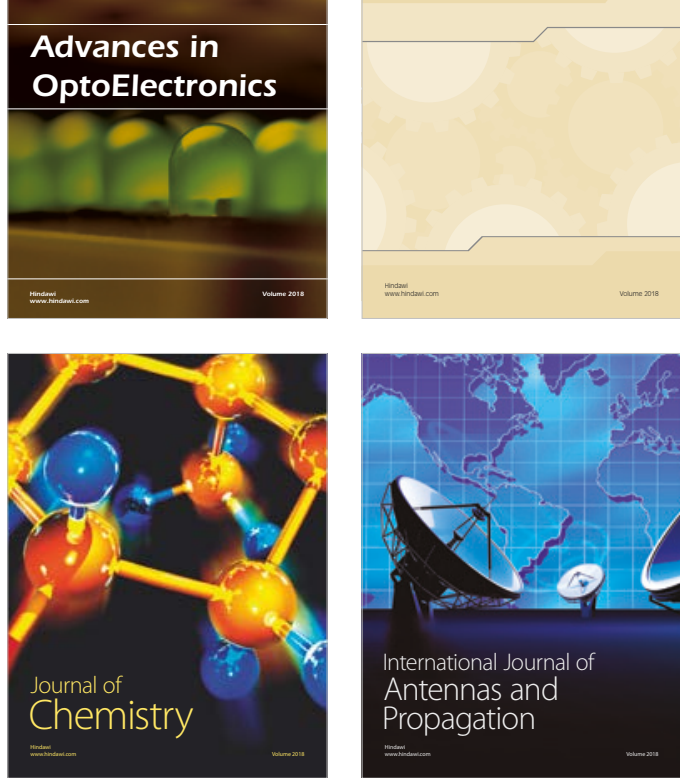

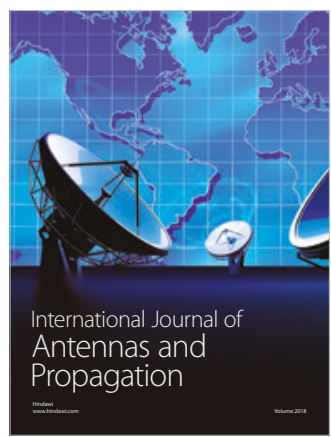

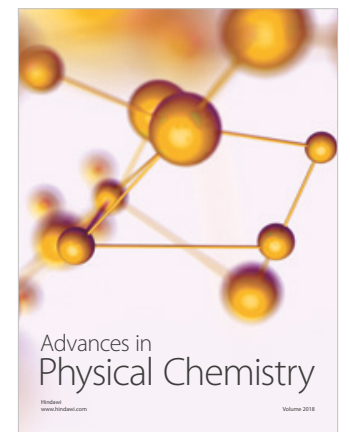

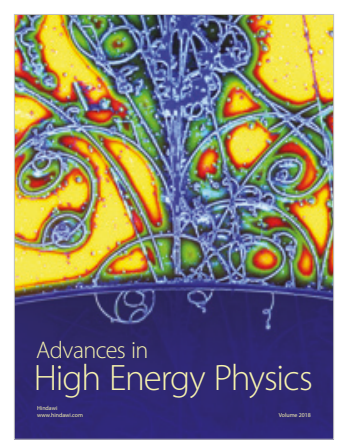

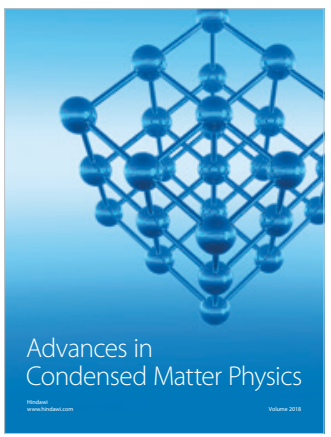

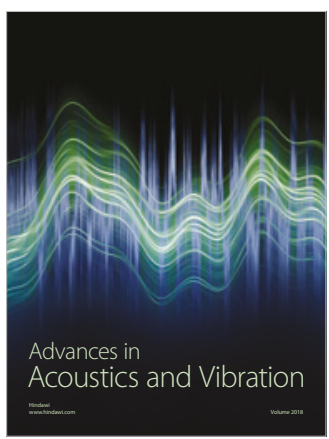

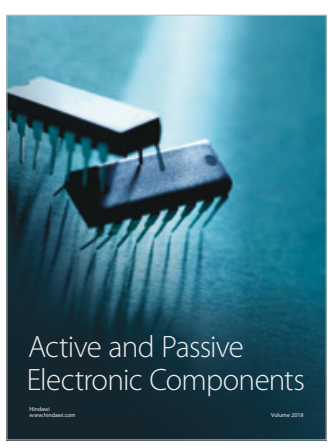
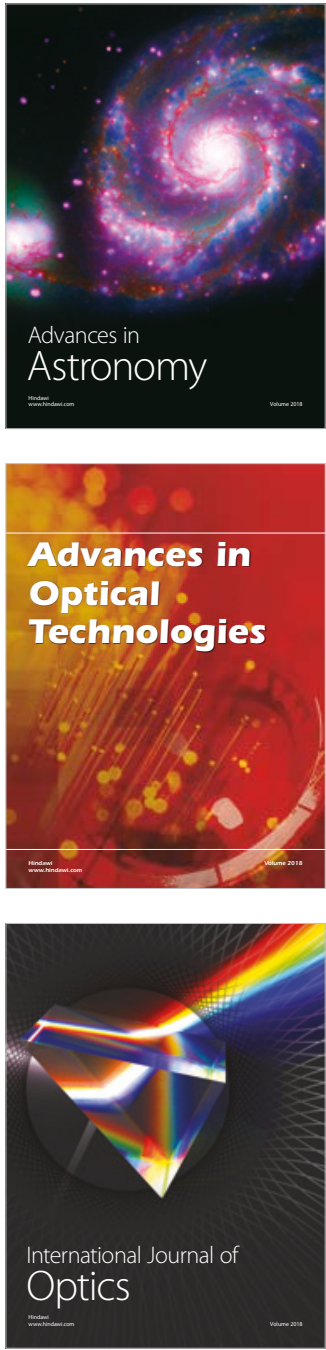